\title{
【Transaction】
}

\section{The Techniques and Origin of Ornamental Gold Silks in Ancient China}

\author{
Xiaorui $\mathrm{Hu}^{* 1}$ and Weidong $\mathrm{Yu}^{* 1, * 2, \text { \# }}$ \\ ${ }^{* 1}$ College of Textiles, Donghua University, Shanghai 201620, China \\ ${ }^{* 2}$ Key Laboratory of Textile Science \&Technology, Ministry of Education, Shanghai 201620, China
}

\begin{abstract}
The ornamental gold silk is a special kind of costly silk fabrics, and it is considered to have a long history in ancient China. This study investigated the origin of the ornamental gold silks and the corresponding gold-decoration techniques, and offered an earlier time for the origin of the ornamental gold silk. Moreover, different gold-decoration techniques were summarized in three categories, i.e. the gold foil method, gold thread method and gold powder method, based on the shape of the processed gold materials. According to the analysis of the complexity of the three techniques, it confirmed that the gold powder method and the gold foil method predated the appearance of the gold thread method, and a query about the existing timeline of gold foil method and gold powder method was proposed.
\end{abstract}

Keywords : Ancient China, Silk, Ornamental Gold, Traditional Technology

(Received 30 January, 2016; Accepted 29 March, 2016)

\section{Introduction}

The ancient China civilization was one of the most splendent urban riverine ancient civilizations in the world, and silks were also considered one of the main contributions of the ancient China. Many archaeological evidences have testified that silk originated from ancient China, and silk fabrics have been produced in China since around $4000 \mathrm{BC}$ [1]. In ancient time, the silk was a generic term of all smooth fabrics made of silk yarns, and it could be classified into Tabby (絹), Gauze (純), Grey Gauze (縞), Leno (羅), Damask (綺), Brocade (錦), Twill damask (綾), Satin (緞), K’o-ssu (緙絲) and Gilt silk (織金) in an approximate chronological order. Innovations in mechanical looms made it possible to produce silks with complicated structures. Similarly, the use of the gold also has a long history, which could go back to 3600 years ago in China [2]. The application of the gold to silks facilitated the development of silk art and civilization dramatically [3]. However, the research concerning the origin of the combination of the gold and silk and the corresponding technique modalities used for ornamental gold silks was rarely reported. Therefore, this paper focuses on the early evidences that gold was applied to the silks and figured out three techniques used for combining the gold and silk in ancient China.

\section{The combination of the silk and the gold in the early stage}

Both of gold and silk were regarded as gorgeous and precious goods in ancient China. As a result, silk textiles were only enjoyed by emperor and aristocracy before Western Han Dynasty [4] (202 BC$8 \mathrm{AD}$ ), and gold has been regarded as significantly valuable materials from time immemorial because of its peculiar qualities. Thus, the combination of the shining gold and the richly decorative silk became the most precious "valuables" in ancient China. It's said that the price of silks from China was as high as the price of gold in Central Asian and Europe from the Two Han Dynasties to Tang Dynasty (618-907 AD) [5] Therefore, decorating gold on the silk makes the silk more valuable and costly. It has been demonstrated that the feudal ruling class was keen on pursuing this kind of gorgeous fabrics and using them as the cloth because this could reveal their wealth and luxury in ancient society [6].

\# corresponding author: Weidong Yu (email: wdyu@dhu.edu.cn) 
The gold, as a kind of precious metal, has also been used throughout the entire civilization history. According to a series of archaeological discoveries and historical research, ample evidence could be employed to prove that gold was firstly used in ancient Egypt and Mesopotamia at least 4000 B.C. on a world scale [7]. The origin of the gold in China may be later than that in ancient Egypt, for instance, the unearthed gold earrings and gold bracelets (Fig. 1) from the “Culture of Lower Xiajiadian” (夏家店下層文 化) and the Siba Culture belonging to Xia Dynasty (21st century - 16th century BC), which may be dated to 3600 years ago. In China, silks appeared approximately 2000 years before the appearance of gold according to the existing information, but the combination of the gold and silk is relatively late, which depends on the making craft of the gold, especially the development of the gold wire's making craft. However, from Xia Dynasty to Shang and Western Zhou Dynasties, the making techniques in terms of gold items developed quickly and a lot of archaeological discoveries during this period could prove the prosperity of the gold process [8].

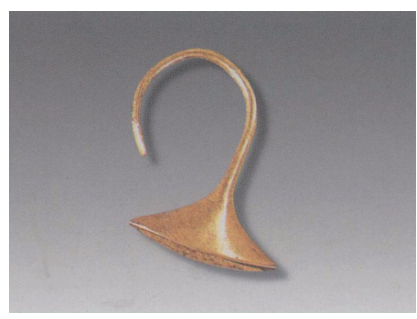

a. Gold earring

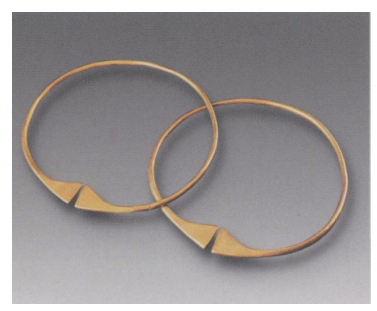

b. Gold bracelet
Fig. 1 Unearthed from Liujiahe village at Pinggu, Culture of Lower Xiajiadian.

Gold can be made into very thin gold foils and gold wires because of its softness and good malleability. In tomb of Yin Ruins in Anyang (Henan, China), the thickness of an unearthed gold foil is 0.010 $( \pm 0.001) \mathrm{mm}$ (i.e. micrometer scale : $1 \sim 100 \mu \mathrm{m})$, which is in concordance with the magnitude of the fiber diameter [7]. Thus it can be deduced that the process technology of the gold in China had reached a certain level at that time and it conformed to the objective condition that gold can be combined with silk dimensionally. As the conclusion of a guess, the gold foils and threads have been introduced into silk fabrics in Shang Dynasty (16th century - 11th century $\mathrm{BC})$.

\section{Techniques of making ornamental gold silks}

Diverse techniques were used in ancient China for making silk fabrics decorated by gold materials. According to the processing properties of the golds, the techniques of making ornamental gold silks were figured out by dividing them into three categories in the present paper, that is, gold foil method, gold thread method and gold powder method. These three methods were explained and analyzed respectively in the following sections.

\subsection{Gold foil method}

The main method for making traditional gold foil is forging. “ Heavenly Creations” (天工開物) [9], a book written by Song Yingxing (1587-1666 AD, Ming Dynasty), described the specific process of making gold foils in ancient China. The text content is shown in Fig. 2 :

The brief meaning of Fig. 2 is as follows :

Firstly, melting the gold and pouring it into a special metal tank to cool the liquid gold to gold bars. After that, reducing the size of gold bar as a $1 \mathrm{~cm}^{2}$ gold leaf. Finally, the gold leaf is beaten so that it becomes gold foil with size of 20 times larger than the original gold leaf based on several processes and thousands of beatings. During the beating, the gold leaves are separated by a special paper named "Wu Jin paper" in order to give full play of the malleability of the gold. When attaching the gold foil onto the silk fabrics, the adhesive (usually use ripe lacquer in ancient) is pasted on the fabric at first. Then the gold foils are put onto the silks with bamboo pliers.

In the early stages, the gold foil was usually used for a number of applications, such as decoration and overlaying or pasting some implements on the surface of them, which could be proved by the discoveries at Erligang Site [7] of the early Shang Dynasty in

“凡金䈃每金七厘造方寸金一千片，粘铺物面，可盖纵横三尺。凡造金䈃， 既成薄片后，包入乌金纸内，竭力挥椎打成…凡纸内打成䈃后，取出乌金纸 内䈃, 覆于其上, 针刀界画成方寸。口中屏息, 手执轻杖, 唾湿而挑起，夹 于小纸之中。以之华物, 先以熟漆布地, 然后粘贴”。

Fig. 2 The text content for specific process of making gold foils from Heavenly Creations. 
Zhengzhou (Henan, China) where some gold foils with a kind of dragon pattern were uncovered, but these foils had fallen off from some implements when they were found. A further example, some gold foils excavated from Anyang Yin Dynasty Ruins [7] were also considered to be attached to wood ware or lacquer work. Thus, it is thought that gold foil method has been used in China for the preparation of gold foils as early as the 10th century BC. From simplex pure gold implements to the implements of the combinations of gold foils and stone, bronze, lacquer, jade and other materials, the Chinese in ancient time created a series of splendid decorative art and artwork by using gold foils. Therefore, putting the gold foils onto the surface of the silk became a natural process. The typical pictures of gold foil together with silks are showed in Fig. 3, where Fig. 3a is a silk blouse printing with gold foils pattern which is from Huang Sheng's grave of the Southern Song Dynasty (1127-1279 AD) in Fuzhou, China, and Fig. 3b shows a piece of printing gold foil silk restored by modern textile artists.

Ancient China, as the main source region of the

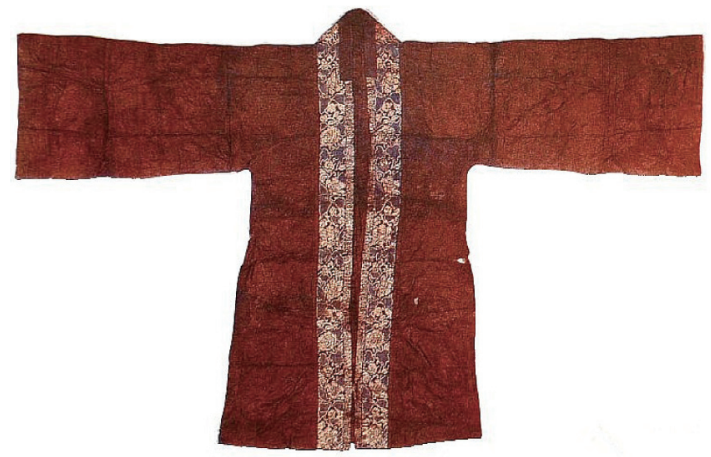

(a) Silk blouse printing with gold foils pattern from Huang Sheng's grave

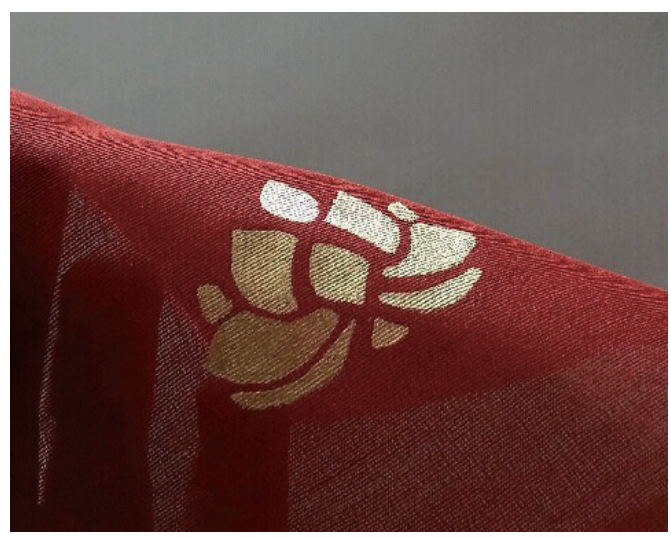

(b) Modern printing gold foil silks

Fig. 3 The detailed pictures of gold foils together with silks. silk in ancient world, has already had corresponding records about silkworm and silk manufacturing in Shang and Zhou Dynasties (11th century-5th century BC) based on some ancient books such as the Book of Songs (詩經), Er Ya (爾雅) and Zhou li (周禮). However, silk ages easily because of its silk fibrin component, and because of this, the silk textiles that were decorated by the gold foils as early as 3000 years ago have disappeared without trace, and only the gold foils still exist at present. The Swedish Bergman [10] dug out an ancient tomb during Eastern Han, and he discovered that there was a red ribbon around an unbleached circular silk pocket (Fig. 4a), and the gold foils were just stuck on the ribbon. Furthermore, there was another beige checkered ribbon covered on an iron mirror (Fig. 4b), and the mark of a row of adherent gold foils was on this beige ribbon. Sylvain [10] , an ancient textile researcher from Sweden, also believed that the technology attaching gold foils to the soft material may derived from ancient China on his research. Thus, synthesizing the point of view of the existing archaeological data, it is thought that the history of adding the gold foil to the silk can be traced to the Eastern Han Dynasty (25-220 AD).

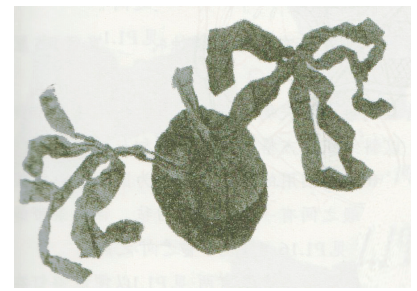

(a) The circular silk pocket and silk ribbons around it

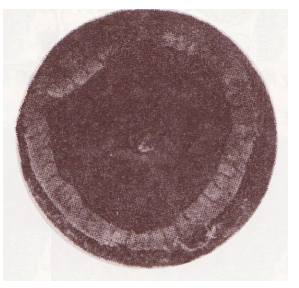

(b) Corroded bronze mirror and the checkered silk ribbon

Fig. 4 Relics unearthed from the Lop Nor Creek No.6 Tomb.

\subsection{Gold thread method}

Gold thread is a kind of yarn that is made of gold or other metals. The gold thread has three forms: (1) Gold wire (made only of metal material) ; (2) Goldwrapped thread (in most case, the core yarn is silk wrapped with a band or strip of high content gold foil); (3) Yarn coated with gold powder. The first and second forms are expounded in detail below. 
(1) Gold wire

Gold wire can be divided into two types according to the thickness: one is "thick gold wire" (0.1 $\sim \mathrm{mm}$ in diameter) and the other is "thin gold wire" (1 100 $\mu \mathrm{m}$ in diameter). Another classification is based on the number of the wire: one is "single gold wire" and the other is "composite gold wire". The single gold wire tends to be thick and it appears earlier as the thick one can be made into the expected shape by hammering directly, in other words, its process technique is relatively easily. This shows that the birth time of the process technique for gold thread strongly depends on the complexity of the technique. The composite gold wire is formed by twisting a number of single gold wires, and the single gold wires can be either thick or thin. Two kinds of molding methods can be used for making thin gold wire. One is called "wire drawing", which employs the approach of tapping or squeezing to draw out the wire; the other is called "shred foil", i.e. cutting the ultrathin gold foil into filaments. Early gold wires in China were generally processed by the approach of the "wire drawing" method. For instance, the aforementioned gold earring and gold bracelet from the "Culture of Lower Xiajiadian" also applied this kind of gold wire [11]. However, the historical testimony about the "wire drawing" of inchoate gold applying to the combination of silk and gold is barely few at present, and this paper mainly focuses on the investigation of the "shred foil" method, and

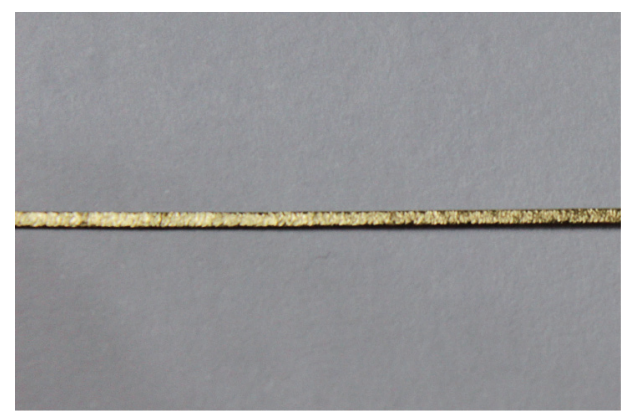

(a) The front of the flat gold

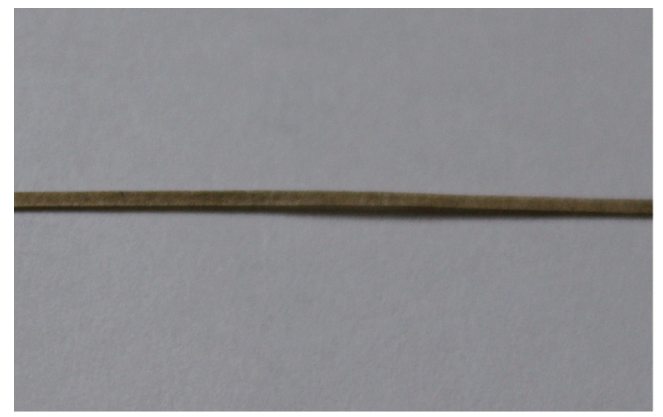

(b) The reverse side of the flat gold

Fig. 5 Photos of the flat gold. apparently, the gold wire is also based on the "foil". It has been found that the "shred foil" didn't appear until the cutting technique of metal foil was created. In the field of textile in ancient China, this kind of gold wire had a new scientific name - “flat gold” (“片金線”) (Fig. 5).

Till now, the making process of the flat gold is similar to that in ancient time, and it could be divided into three steps in details: firstly, immersing the bamboo paper in water and making it wet, and then puting the gold foil on the bamboo paper after brushing a layer of fish gelatin on it. Secondly, calendaring the aforesaid paper gold foils on a tailormade board (generally using wild pear wood) with cobbles in order to improve the surface gloss of gold foils. Finally, cutting the gold foil into fine long shreds with a mechanical cutting machine to make the "flat gold".

(2) Gold-wrapped thread

Chen Weiji (1902-1984 AD) [12], a famous textile technologist, has summed up the craftsmanship of gold-wrapped thread for textiles used in ancient China in considerable detail in his book entitled "History of Science and Technology of China Textile (Ancient)". The forming steps of the gold-wrapped thread can be simply summarized as below: Firstly, making a fibrous core of silk, linen, cotton or other yarns, and then winding the gold strip around the fibrous core in a spiral shape to make a gold-wrapped thread (the model of gold-wrapped thread is shown in Fig. 6). For some aspects, more emphasis should be put on, for example, when preprocessing and dyeing the silk or cotton yarns, the primary hue should be consistent with the gold color [13].

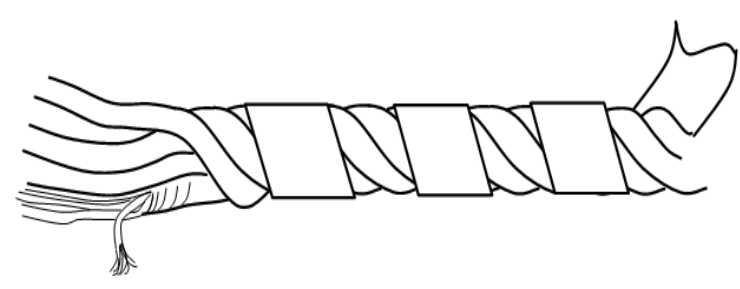

Fig. 6 The model of gold-wrapped thread.

According to the existing archaeological data, the earliest gold-wrapped threads found in China are from Sinkiang. One is from the Wei-Jin Mural Tomb Hexi Corridor region and another is from the Shanpula Tomb in Luopu County. It is estimated that both of them were made about 1660-1700 years ago [14]. In addition, the gold-wrapped threads that were unearthed from Famen Temple of Xian are also 
antique (about 1400 years ago). Three microscopic topography pictures of the early unearthed goldwrapped threads from Wei-Jin Mural Tomb, Shanpula Tomb and Famen temple are shown in Fig. 7 [15].

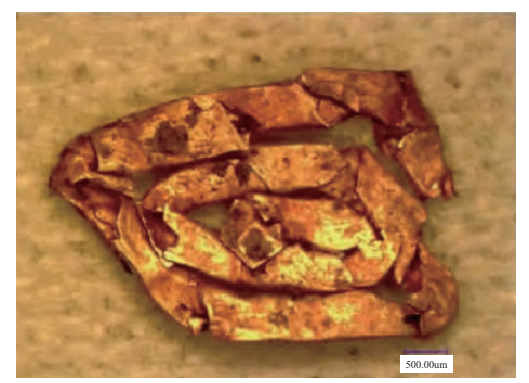

(a) Composite gold thread from the Tomb of Sixteen Countries, Xinjiang

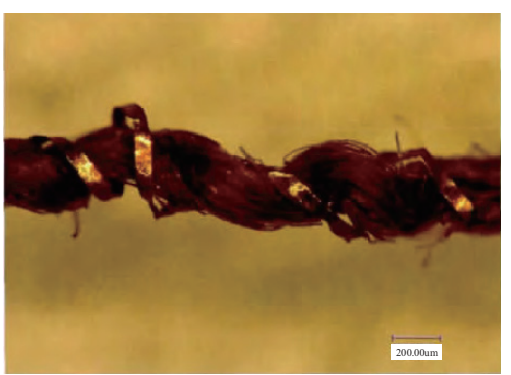

(b) Composite gold thread from Shanpula site, Xinjiang

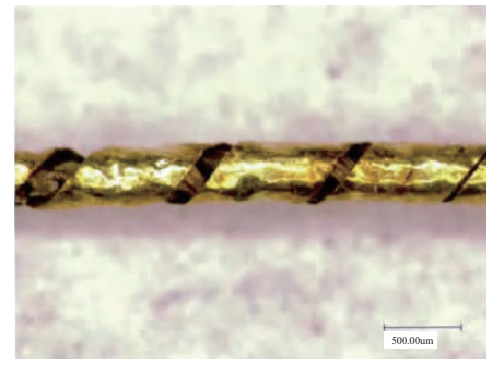

(c) Composite gold thread from Famen temple, Xian

Fig. 7 Microscopic topography of the early unearthed composite gold threads in China.

\subsection{Gold powder method}

There is no doubt that the basic of the gold powder method is the manufacture of the gold powder, and what the most important is that the gold powder should reach the micrometer size, i.e. $1 \sim 10 \mu \mathrm{m}$. Once the gold powder is ready, and then the next step is to choose a suitable binder and to mix up the gold powder with the binder. The last step is to put the mixture onto the surface of the silk yarns or fabrics through the printing, painting or dipping extrusion methods. Obviously, the gold powder method used on yarns is much more complex than the method used on fabrics. Meanwhile, the historical records of silk yarns coated with gold powders are very rare, thus the paper mainly discussed the techniques of printing or painting gold powders to the silk fabrics. It is the binder that keeps the gold powder fixed on the surface of the silks, so the implementation of this method depends on the discovery and employment of binder which is supple and firm. In consequence, it becomes more difficult not only for using the gold powder method but also for applying the gold foil (glued) method to decorate the silk, but it is the difficulty that makes the textiles decorated with gold powder precise. Unfortunately, the silk fabrics decorated with gold powder usually have bad softness, and the producing of these fabrics also needs the professional technicians. Therefore, in later generations, the ancient Chinese turned their attention to the approach of applying thin gold wire and gold-wrapped thread to the silks.

In ancient China, gold powder was used for a lot of fields including medicine, paint, printing paper and silk. The origin and development of gold powder occurs later than that of gold foil, but there were many references about native gold powder in ancient Chinese books. In the early stage, the technology for making gold powder probably involved grinding gold on a special stone. Later, more refined techniques including mechanical method and chemical method were developed all at once in the 2nd century AD. The mechanical method can be subdivided into grinding method, abrasion method and mechanical comminution. Hu Gangzi, an alchemist in Eastern Han Dynasty (25-220 AD), invented a chemical method for making gold powder in the 2nd century AD. This method was described in 'Chu Jin Kuang Tu-Lu' (出金 礦圖錄) written by Hu Gnagzi [16]. The specific process of this method is described as following: firstly making the gold into liquid amalgam after dissolving in the mercury. Then puting the mixture together with salt and grind them adequately. When the gold disperses on the surface of the salt, evaporating the mercury. After cleaning with water, the salt is gone, and we get the fine gold powder finally.

In 1972, a piece of gold and silver mud flame (Fig. 8), which had a history of 2100 years was unearthed from Tomb 1 at Mawangdui Site [17]. It should be the earliest silk fabric that was metalized with gold powder. During 1975, archaeologists found female dresses were printed pattern with gold powder at Huang Sheng's grave in Fuzhou, China [18] These clothes were representative of printing silk 


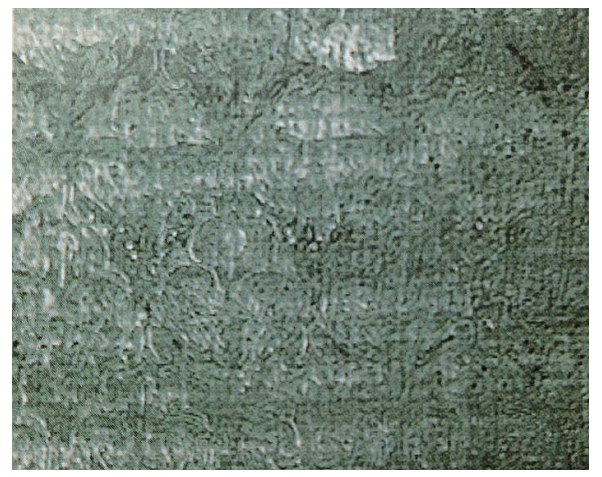

(a) Physical picture of the gold and silver mud flame

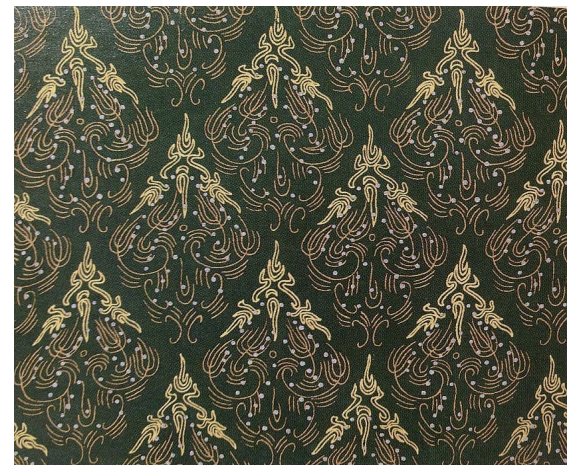

(b) Reconstructed picture of the gold and silver mud flame

Fig. 8 Gold and silver mud flame from Tomb 1 at Mawangdui Site.

with gold powder in the Southern Song Dynasty (1127-1279 AD).

\subsection{Time sequence of three methods}

According to on-hand unearthed reference materials, the three techniques of making silk decorated in gold were generally ordered in time sequences, as shown in formula 1: ( $t$ expresses the length of time from now)

$$
t_{\text {gold powder }}>t_{\text {gold foil }}>t_{\text {gold thread }}
$$

It historically indicates that the gold powder method is the first method used as silk-decoration techniques, and the gold thread is developed relatively later than the other two methods. However, based on the complexity of the techniques and the preparation time spent on technique process, we can also assume a time order in terms of the time when these three methods apply to the silk fabrics, as following :

$$
t^{\prime} \text { gold foil } \geq t^{\prime} \text { gold powder }>t^{\prime} \text { gold thread }
$$

Where " $t$ ' gold foil $>t$ ' gold powder", this is because gold powder method is developed based on the discovery and application of binders, distribution of gold powder in binders and the development of grinding and painting technologies. The binder should be soft solids and have high cohesion and the performance of rapid solidification. However, the gold foil is mainly made by forging, which tends to be easier in technique process and is recognized and used earlier than the method for making powder. For $t^{\prime}$ gold foil $=t^{\prime}$ gold powder, it can be explained that the time consuming and difficulty between grinding gold powders and hammering gold foils are quite similar. Therefore, the time relationship is expressed as $t^{\prime}$ gold foil $\geq t^{\prime}$ gold powder, Equation (1) matches equation (2) well, which also demonstrates that the techniques of making gold thread from gold are very complex and elaborate, and the development of the processing-gold techniques is a major factor that affects the application of the different silkdecoration techniques. In addition, it is also easier to make gold foil from gold and then make gold foil into gold powder as shown in equation (2), however, the equation (1) shows a different order. The differences between equation (1) and equation (2) may result from a fact that gold powder method appears before appearance of gold foil method. Therefore, we conclude a view that it is not accurate to judge timeline only based on the unearthed objects.

\section{Conclusions}

The paper investigated the inchoate combination of the gold and silk, and proposed that the ornamental gold silks may have come into being as early as Shang Dynasty, almost three thousand years ago. Different techniques used for making ornamental gold silks were analyzed, and three gold-decoration techniques, i.e. gold foil method, gold thread method and gold powder method, were classified based on the shape of the processed gold materials. Also, the time sequences of the development of the three techniques were discussed according to the on-hand unearthed reference materials and their technique complexity, respectively. It confirmed that the gold powder method and the gold foil method predated the appearance of the gold thread method, and a query about the timeline of gold foil and gold powder method is proposed, which needs to investigate in further work.

\section{Acknowledgements}

The authors are grateful to Mr. Wen Jin (brocade artist from Nanjing, China) for providing the photos of flat gold threads. The financial support of the Fundamental Research Funds for the Central Universities (CUSF-DH-D-2016020) is also appreciated. 


\section{References}

1. H. Nengfu, C. Juanjuan, China Textile Press, $2-5$ (2002).

2. J. HJ, Ko SH, Journal of the Korean Society of Costume. 56(4), 82-95 (2006).

3. Good IL, Kenoyer JM, Meadow RH, Archaeometry. 51(3), 457-66 (2009).

4. X. Nai. Archaeology, 2, 12-27 (1972).

5. Watt J C Y, Wardwell A E, Metropolitan Museum of Art, 6-10 (1997).

6. Bunker E C, Artibus Asiae, 27-50 (1993).

7. A. Zhimin, A. Jianyuan, Acta Archaeologica Sinica, 3, 291-310 (2008).

8. Hodges $\mathrm{H}$, Technology in the ancient world. Barnes \& Noble Publishing, 135-167 (1992).

9. S. Yingxing, Heavenly Creations, Shanghai, Shanghai ancient books publishing house, 99-100 (2013).
10. W. Bergman, Archaeological Researches in Xinjiang, Urumchi, Volksverlag Xinjiang, 157-173 (2013).

11. Y. Jinjing, Z. Xiande, Cultural Relics, 11, 1-8 (1977).

12. C. Weiji. Science Press, 130-137 (1984).

13. X. Zhengxing, Journal of Shanghai Textile Institute of Technology, 2, 83-89 (1980).

14. L. Zhiyong, Relics and Museology, 1, 70-74 (2014).

15. D. Xiaojuan, G. Jinlong, B. Ke, Y. Junchang, Sciences of Conservation and Archaeology, 3, 13-18 (2014).

16. Z. Huaizhi, N. Yuantao, Gold Bulletin, 33(3), 103105 (2000).

17. Shanghai Textile Science Research Institute, Cultural Relics Press, 108-111 (1980).

18. C. Wei, Weaving, Embroidery, Wenhui Press, 948 (1996). 